\title{
Rendimiento y calidad de Avena sativa asociada con Vicia sativa en la región puna del Perú
}

\author{
Yield and quality of Avena sativa associated with Vicia sativa in the \\ puna region of Peru
}

\author{
Alberto Arias A. ${ }^{1}$, Juancarlos Cruz L. ${ }^{2}$, Cesar Pantoja A. ${ }^{3}$, José Contreras P., ${ }^{4,5}$, \\ Melina Lopez R. ${ }^{1}$
}

\section{Resumen}

\begin{abstract}
El estudio se realizó en el Centro Experimental Alpaicayán, Universidad Nacional Daniel Alcides Carrión, Pasco, Perú, con el objetivo fue determinar la producción forrajera y calidad nutricional de la avena asociada con vicia. Se determinó la producción forrajera en contenidos de materia verde (MV), materia seca (MS) y el porcentaje de materia seca $(\% \mathrm{MS})$. Para el valor nutricional se determinaron la proteína cruda $(\% \mathrm{PC})$, fibra detergente neutro $(\% \mathrm{FDN})$, fibra detergente ácido $(\% \mathrm{FDA})$, calcio $(\% \mathrm{Ca})$ y fósforo $(\% \mathrm{P})$. Los tratamientos fueron T-1 (avena criolla, $150 \mathrm{~kg} / \mathrm{ha}$ ), T-2 (avena Mantaro 15, $110 \mathrm{~kg} / \mathrm{ha}$ ), T3 (avena Mantaro 15 más vicia, 110 y $30 \mathrm{~kg} / \mathrm{ha}$ ) y T-4 (avena criolla más vicia, 150 y 30 $\mathrm{kg} / \mathrm{ha}$ ). La producción de MV indicó que T-1 y T-4 fue similar, pero significativamente superior a T-2 y T-3 $(\mathrm{p}<0.05)$; sin embargo, sin diferencias significativas en producción de MS entre tratamientos. T-4 obtuvo mayor contenido de \% PC, T-2 y T-4 presentaron los
\end{abstract}

${ }^{1}$ Facultad de Ciencias Agropecuarias, Universidad Nacional Daniel Alcides Carrión, Cerro de Pasco, Perú

${ }^{2}$ Dirección de Supervisión y Monitoreo en las Estaciones Experimentales Agrarias, Instituto Nacional de Innovación Agraria, Lima, Perú

${ }^{3}$ Carrera Profesional de Zootecnia, Universidad Nacional Daniel Alcides Carrión, Cerro de Pasco, Perú

${ }^{4}$ Facultad de Ciencias Agrarias, Universidad Nacional de Huancavelica, Huancavelica, Perú

${ }^{5}$ E-mail: jose.contreras@unh.edu.pe

Recibido: 10 de enero de 2021

Aceptado para publicación: 23 de agosto de 2021

Publicado: 27 de octubre de 2021

CLos autores. Este artículo es publicado por la Rev Inv Vet Perú de la Facultad de Medicina Veterinaria, Universidad Nacional Mayor de San Marcos. Este es un artículo de acceso abierto, distribuido bajo los términos de la licencia Creative Commons Atribución 4.0 Internacional (CC BY 4.0) [https:// creativecommons.org/licenses/by/4.0/deed.es] que permite el uso, distribución y reproducción en cualquier medio, siempre que la obra original sea debidamente citada de su fuente original 
mayores rendimientos de \%FDN y \%FDA. T-4 presentó el mayor contenido de \%P, en tanto que no hubo diferencias entre tratamientos para el contenido de $\% \mathrm{Ca}$. Se concluye que el tratamiento Mantaro 15 +vicia tiene una mejor producción forrajera y es de mejor calidad nutricional en referencia a los otros tratamientos.

Palabras clave: avena, forraje, valor nutricional, región puna, pastos anuales

\section{AbSTRACT}

The study was conducted at the Alpaicayan Experimental Centre, Daniel Alcides Carrión National University, Pasco, Peru, with the objective of determining the forage production and nutritional quality of oats associated with vetch. Forage production was determined in green matter content (GM), dry matter (DM) and percent of dry matter $(\% \mathrm{DM})$. For the nutritional value, crude protein $(\% \mathrm{CP})$, neutral detergent fibre $(\% \mathrm{NDF})$, acid detergent fibre $(\% \mathrm{ADF})$, calcium $(\% \mathrm{Ca})$ and phosphorus $(\% \mathrm{P})$ were determined. The treatments were T-1 (Creole oat, $150 \mathrm{~kg} / \mathrm{ha}$ ), T-2 (Mantaro 15 oat, $110 \mathrm{~kg} / \mathrm{ha}$ ), T-3 (Mantaro 15 oat plus vetch, 110 and $30 \mathrm{~kg} / \mathrm{ha}$ ) and T-4 (creole oat plus vetch, 150 and $30 \mathrm{~kg} / \mathrm{ha}$ ). GM production indicated that $\mathrm{T}-1$ and $\mathrm{T}-4$ were similar but significantly greater than $\mathrm{T}-2$ and T-3 $(\mathrm{p}<0.05)$; however, without significant differences in DM production between treatments. T-4 obtained the largest content of $\% \mathrm{PC}, \mathrm{T}-2$ and T- 4 presented greater yields of $\% \mathrm{NDF}$ and $\% \mathrm{ADF}$. T-4 presented the highest content of $\% \mathrm{P}$, while there was no difference between treatments for the content of $\% \mathrm{Ca}$. It is concluded that the Mantaro $15+$ vetch treatment has a better forage production and is of better nutritional quality in relation to the other treatments.

Key words: oats, forage, nutritional value, puna region, annual pastures

\section{INTRODUCCIÓN}

La avena (Avena sativa $\mathrm{L}$ ) es una importante planta productora de forraje y la más difundida y de mucha importancia para la región Puna, debido a su alta digestibilidad, buena cantidad de energía metabolizable y fibra de mejores cualidades que otros cereales; además, el grano presenta elevada cantidad y calidad de proteínas, carbohidratos, minerales, grasas y vitamina B (Noli et al., 2006; Ramírez et al., 2013; Zapana et al., 2014). Es una gramínea anual que produce altos volúmenes de forraje en la época de lluvia (20-30 t/ha de materia verde y entre 2.4-3.2 $\mathrm{t} /$ ha de materia seca - MS) y con contenidos de proteína total (PT) entre 7.6 y $10.2 \%$, fibra detergente neutro (FDN) entre 34.8 y $42.8 \%$ y alta cantidad de energía meta- bolizable (12.6-14.1 EM MJ/kg/MS) para la etapa fisiológica de grano lechoso (Noli et al., 2006; Arias, 2015).

La avena forrajera es un cultivo de adaptabilidad a distintas condiciones ambientales (2500-4200 msnm), se puede establecer en suelos con textura ligera a media (franco a franco limoso) a ligeramente arenosa (franco arenoso), sin problemas de drenaje y con un $\mathrm{pH}$ ligeramente ácido a neutro (5-7). La conservación puede darse como heno o ensilado a fin de almacenar alimento para la época de estiaje (mayo-setiembre) (Noli et al., 2006; Ramírez et al., 2013; Villareal, 2013; Arias, 2015), permitiendo asegurar la producción del ganado en la época seca donde hay escasez de pastos naturales (Contreras et al., 2013). 
Vicia sativa es una de las leguminosas forrajeras anuales más importantes a nivel mundial, debido a sus múltiples usos y alta calidad nutricional (Kim et al., 2015). Las leguminosas tienen capacidad para desarrollar nódulos en sus raíces y fijar nitrógeno en simbiosis con rhizobios compatibles (Graham y Vance, 2000), que es utilizado por la planta huésped o por cultivos asociados (Graham y Vance, 2000), siendo la tasa de fijación de nitrógeno de 1 a 2 kg/ha/día (Giller, 2001), con el cual evita la degradación de los suelos y al mismo tiempo mejora la calidad de suelos degradados (Cocks, 1995).

En este contexto, los productores individuales, comunidades campesinas, cooperativas comunales y demás entes dedicados a la producción pecuaria de ovinos, vacunos y camélidos en la región Puna, viene incorporando la siembra de avena forrajera para la alimentación de sus animales por ser fácil de sembrar y conservarse sin mayor esfuerzo. Sin embargo, son pocos los estudios realizados en la determinación y análisis de la producción de forraje y la calidad nutricional de este cultivo, tanto en la siembra del cultivo solo como asociado con vicia (Vicia sativa). Por tanto, el objetivo de este trabajo de investigación fue determinar la producción de forraje y la calidad nutricional de Avena sativa sola o asociada con Vicia sativa bajo condiciones de altura a $4127 \mathrm{msnm}$ en región Puna de Perú.

\section{Materiales y Métodos}

El estudio se realizó en el Centro Experimental Alpaicayán de la Universidad Nacional Daniel Alcides Carrión, ubicado en el distrito de Vicco, provincia y departamento de Pasco, a una altitud de $4127 \mathrm{msnm}$, definida como una ecorregión puna húmeda seca (Britto, 2017). La vegetación predominante en la zona es Stipa ichu, Calamagrostis antoniana, Calamagrostis vicunarum y Festuca sp. El escenario edáfico comprende suelos profundos con una clase textural de franco arenoso con un $\mathrm{pH}$ de 5.15 y contenidos de materia orgánica de $4.4 \%$; asimismo, la concentración de fósforo en el suelo es de $12.8 \mathrm{ppm}$ y de potasio de $39 \mathrm{ppm}$ (Arias, 2015).

La preparación del terreno se realizó con maquinaria agrícola. Se inició con el arado de discos a una profundidad de $30 \mathrm{~cm}$ con la finalidad de aflojar la tierra, seguido por la rastra cruzada a fin de mullir el terreno (Chávez y Gómez, 1999; Noli et al., 2010). Se trabajó con cuatro parcelas de $4420 \mathrm{~m}^{2}$, donde se instalaron al azar los cuatro tratamientos según la densidad de siembra (Cuadro 1). Se consideraron las recomendaciones de Cazares (1999) que menciona que la siembra con mayor cantidad de semilla por hectárea se da cuando las semillas son más pesadas y la siembra se da en época tardía (caso del estudio). La siembra se hizo al voleo el 25 de noviembre de 2015, utilizando semillas en etapa fisiológica de grano lechoso provenientes del Instituto Nacional de Innovación Agraria (INIA). La siembra se hizo sin hacer alguna corrección en los suelos.

Para determinar la producción de forraje en materia verde (MV) en $\mathrm{kg} \mathrm{ha}^{-1}$ en cada parcela se lanzó al azar por 10 ocasiones un cuadrante de $1 \mathrm{~m}^{2}$. Se cortó y pesó todas las plantas (tallo, hoja y granos) que se encontraban dentro del cuadrante. La MS se calculó a partir de las muestras obtenidas, una vez que fueron secados al aire libre durante cinco días a una temperatura ambiente media de $17{ }^{\circ} \mathrm{C}$ y $54 \%$ de humedad relativa, completándose el secado en estufa a $60^{\circ} \mathrm{C}$ durante 48 horas. El material vegetal seco fue pesado.

El análisis de calidad nutricional se determinó a partir de las muestras secas. Estas fueron trituradas y molidas en un molino Wiley con malla de $1 \mathrm{~mm}$ en el Laboratorio de Evaluación Nutricional de Alimentos de la Universidad Nacional Agraria La Molina, en Lima. Se analizaron las siguientes variables: proteína cruda (PC), fibra detergente neutro 
Cuadro 1. Tratamientos (T) del estudio con Avena sativa sola o asociada con Vicia sativa

\begin{tabular}{clc}
\hline & Especie & $\begin{array}{c}\text { Densidad de siembra } \\
\text { (kg/área de ensayo) }\end{array}$ \\
\hline T-1 & Avena criolla $(150 \mathrm{~kg} / \mathrm{ha})$ & 67.8 \\
$\mathrm{~T}-2$ & Avena Mantaro $15(110 \mathrm{~kg} / \mathrm{ha})$ & 48.8 \\
$\mathrm{~T}-3$ & Avena Mantaro $15(110 \mathrm{~kg} / \mathrm{ha})+$ Vicia $(30 \mathrm{~kg} / \mathrm{ha})$ & $44.2-12.5$ \\
$\mathrm{~T}-4$ & Avena criolla $(150 \mathrm{~kg} / \mathrm{ha})+\mathrm{Vicia}(30 \mathrm{~kg} / \mathrm{ha})$ & $48.6-12.5$ \\
\hline
\end{tabular}

Cuadro 2. Producción de forraje de parcelas sembradas con Avena sativa sola o asociada con Vicia sativa

\begin{tabular}{lcccc}
\hline \multirow{2}{*}{ Variable } & Criolla & Mantaro15 & $\begin{array}{c}\text { Criolla } \\
\text { vicia }\end{array}$ & $\begin{array}{c}\text { Mantaro15 } \\
+ \text { vicia }\end{array}$ \\
\cline { 2 - 5 } & $2.53^{\mathrm{a}}$ & $1.94^{\mathrm{b}}$ & $1.87^{\mathrm{b}}$ & $2.04^{\mathrm{ba}}$ \\
\hline Materia verde $\left(\mathrm{kg} / \mathrm{m}^{2)}\right.$ & $0.88^{\mathrm{a}}$ & $0.72^{\mathrm{ba}}$ & $0.69^{\mathrm{b}}$ & $0.75^{\mathrm{ba}}$ \\
Materia seca $\left(\mathrm{kg} / \mathrm{m}^{2}\right)$ & $35.28^{\mathrm{a}}$ & $35.87^{\mathrm{a}}$ & $36.72^{\mathrm{a}}$ & $36.28^{\mathrm{a}}$ \\
\hline Materia seca $(\%)$ & &
\end{tabular}

a,b Letras diferentes en cada fila indican diferencias significativas entre tratamientos $(p<0.05)$

(FDN), fibra detergente ácido (FDA), calcio (Ca) y fósforo $(\mathrm{P})$, mediante el análisis micro Kjeldahl (Nx6.25) (AOAC, 2005). Para el análisis estadístico se utilizó un diseño completamente al azar, aplicando la prueba de LSD al 5\% para analizar las diferencias entre las medias.

\section{Resultados y Discusión}

\section{Producción de Forraje}

En el Cuadro 2 se presentan las comparaciones de medias para las variables $\mathrm{MV}$, MS y el porcentaje de materia seca (\%MS) de rendimiento forrajero $\left(\mathrm{kg} / \mathrm{m}^{2}\right)$ de las dos variedades de avena y sus asociaciones con vicia. La avena criolla tuvo mayor contenido de forraje en comparación a las demás variedades. De otra parte, no se observaron diferencias significativas en \%MS entre tratamientos.

La variedad con mayor biomasa forrajera $\left(\mathrm{kg} / \mathrm{m}^{2}\right)$ fue la avena criolla (T1), aunque sin diferencias significativas con $\mathrm{T} 4$ (Mantaro15 +Vicia). De otra parte, no hubo diferencias significativas entre T2, T3 y T4. En el Cuadro 3 se presentan valores reportados por diversos autores, muchos de los cuales no concuerdan con los resultados del presente estudio. Estas diferencias se pueden atribuir a las condiciones ambientales en las que fueron evaluadas, pudiendo de esa manera ser un indicativo de que la altitud y las 
condiciones atmosféricas en que son sembradas influyen en la producción y biomasa forrajera de este producto.

No se encontraron diferencias significativas entre tratamientos para \%MS. Los resultados del estudio se encuentran entre los promedios de 28.9-39.4\% MS reportados por Noli et al. (2004a). Sin embargo, son superiores al $17.3 \%$ MS para avena criolla reportado por Shin et al. (1999) con la planta en estado de cosecha. Para la avena Mantaro 15 y Mantaro 15 + vicia se obtuvo 35.87 y $36.28 \% \mathrm{MS}$, respectivamente, valores que superan al 17.32 y $28.04 \%$ MS (proporción 50/50) obtenido por Contreras et al. (2020). Los porcentajes de contenido de MS pueden ser diferentes, pero dependen de factores como la fecha de cosecha, condiciones ambientales y tipos de suelos en que estos son sembrados; sin embargo, el momento óptimo de cosecha recomendado en el estado de grano lechoso, ya que en ese momento todas las variables tanto productivas y de calidad nutricional se encuentran en su mejor proporción (Cortes y Silva, 1995; Dumont et al., 2005; Ramírez et al., 2013).

\section{Valor Nutricional}

En la comparación nutricional se presentaron efectos significativos $(\mathrm{p}<0.05)$ en PC, FDN y FDA y P entre tratamientos, mas no en el contenido de $\mathrm{Ca}$ (Cuadro 3). Los contenidos de PT se encuentran entre los intervalos reportados por Salmerón et al. (2003), Florián (2006) y Arias (2015) con valores entre 6.8 a $12.4 \%$ de PT para avenas forrajeras. Otros valores reportados para PT han sido de $69.7 \mathrm{~g} / \mathrm{kg}^{-1} \mathrm{DM}$ de PC (Caballero et al., 1995) para avena criolla, $100.8 \mathrm{~g} / \mathrm{kg}$ DM de PC en asociado de ensilado avena + vicia (Garduño et al. (2009).

El contenido de PC en T-4 (Avena Mantaro 15 + vicia) mostró un resultado mayor $(p<0.05)$ en comparación a los demás tratamientos, lo cual va en línea con el $7.43 \%$ de PC (proporción 50:50) y 8\% para avena criolla reportado por Contreras et al. (2020) y Shin et al. (1999), respectivamente. Estas diferencias pueden atribuirse a factores como la calidad de semilla, suelo, estado fenológico (Oliver et al., 2004; Dumont et al., 2005) у sobre todo las condiciones ambientales que influyen de manera significativa sobre la composición química del forraje, aun dentro de la misma variedad de avena y su asociación (Espitia et al., 2012; Ramírez et al., 2013). Además, los contenidos de PC dependen mayormente de la cantidad de grano presente en la planta, mientras que el del follaje (hojas y tallos) solo representa niveles de 4 a 5\% (Ramírez et al., 2013).

El contenido de FDN fue significativamente mayor en T-2 y T4 en comparación con T-1 y T-3 (p<0.05) (Cuadro 3). Estos resultados fueron similares a los obtenidos por Shin et al. (1999) y Arias (2015) en la variedad criolla y Mantaro 15, respectivamente, quienes encontraron valores de 37.8 y $34.8 \%$. Asimismo, Johnston et al. (1999), Coblentz et al. (2000) y Salmerón et al. (2003) encontraron valores de 45.0, 62.4 y $62.2 \%$, siendo estos valores mayores a los obtenidos en el presente estudio. Los contenidos de FDN dependen mucho de la calidad genética y del estado de madurez de la planta (Ramírez et al., 2013). La NRC (2001) menciona que la dieta de rumiantes (ovinos, vacunos y camélidos) debe contener entre 25 a $35 \%$ de FDN para asegurar el buen funcionamiento del rumen, favorecer la producción de saliva y mantener un $\mathrm{pH}$ de 6.2-6.8 para una correcta actividad celulítica.

Los contenidos de FDA mostraron diferencias significativas entre tratamientos $(\mathrm{p}<0.05)$, siendo los valores de T-2 y T-4 significativamente superiores respecto a $\mathrm{T}-1$ y T3 (Cuadro 4). Estos resultados son ligeramente mayores a los reportados por Arias (2015), con valores para la avena Criolla y Mantaro 15 de 19.9 y $16.1 \%$, respectivamente. Asimismo, Shin et al. (1999) reportó 16.3\% de FDA para avena criolla, valor similar al del presente estudio. No obstante, estos resultados son inferiores con relación a los hallados por Johnston et al. (1999), Coblentz et 
Cuadro 3. Producción de forraje verde y de materia seca de Avena sativa sola o asociada con Vicia sativa reportada en la literatura científica

\begin{tabular}{lccl}
\hline & Forraje verde & Materia seca & Referencia \\
\hline Avena criolla & $3.3-2.3 \mathrm{~m}^{2}$ & $1.2 \mathrm{~m}^{2}$ & Lithourgidis et al. (2006) \\
Avena criolla & $1.3 \mathrm{~kg} / \mathrm{m}^{2}$ & $0.31 \mathrm{~kg} / \mathrm{m}^{2}$ & Arias (2015) \\
Avena criolla + vicia & $1.87 \mathrm{~kg} / \mathrm{m}^{2}$ & $7.12 \mathrm{~kg} / \mathrm{m}^{2}$ & Ansar et al. $(2010)$ \\
Avena criolla + vicia & $1.63 \mathrm{~kg} / \mathrm{m}^{2}$ & & Espinoza et al. (2018) \\
Avena criolla + vicia & $0.43 \mathrm{~kg} / \mathrm{m}^{2}$ & & Caballero et al. $(1995)$ \\
Avena criolla + vicia & $0.80 \mathrm{~kg} / \mathrm{m}^{2}$ & & Flores et al. (2016) \\
Avena Mantaro15 & $2.08 \mathrm{~kg} / \mathrm{m}^{2}$ & $0.64 \mathrm{~kg} / \mathrm{m}^{2}$ & Noli et al. $(2004 \mathrm{a})$ \\
Avena Mantaro15 & $6.33 \mathrm{~kg} / \mathrm{m}^{2}$ & $3.04 \mathrm{~kg} / \mathrm{m}^{2}$ & Noli et al. $(2004 \mathrm{~b})$ \\
Avena Mantaro15 & & $0.23 \mathrm{~kg} / \mathrm{m}^{2}$ & Shin et al. $(1999)$ \\
Avena Mantaro15 & $2.2 \mathrm{~kg} / \mathrm{m}^{2}$ & $0.30 \mathrm{~kg} / \mathrm{m}^{2}$ & Arias (2015) \\
Avena Mantaro $15+$ & $2.98 \mathrm{~kg} / \mathrm{m}^{2}$ & & Contreras et al. (2020) \\
vicia & & & \\
\hline
\end{tabular}

Cuadro 4. Comparación de medias de variables nutricionales de Avena sativa sola o asociada con Vicia sativa

\begin{tabular}{lcccc}
\hline & \multicolumn{4}{c}{ Tratamientos } \\
\cline { 2 - 5 } Variable & Criolla & Mantaro 15 & $\begin{array}{c}\text { Criolla }+ \\
\text { Vicia }\end{array}$ & $\begin{array}{c}\text { Mantaro15 } \\
+ \text { Vicia }\end{array}$ \\
\hline $\begin{array}{l}\text { Proteína cruda (PC\%) } \\
\text { Fibra detergente neutra }\end{array}$ & $6.54^{\mathrm{b}}$ & $6.02^{\mathrm{c}}$ & $6.55^{\mathrm{b}}$ & $8.28^{\mathrm{a}}$ \\
$\begin{array}{l}\text { FDN\%) } \\
\text { Fibra detergente ácida }\end{array}$ & $36.25^{\mathrm{b}}$ & $38.62^{\mathrm{a}}$ & $35.99^{\mathrm{b}}$ & $39.39^{\mathrm{a}}$ \\
$\begin{array}{l}\text { FDA\%) } \\
\text { Calcio (Ca\%) }\end{array}$ & $19.16^{\mathrm{b}}$ & $20.16^{\mathrm{a}}$ & $19.21^{\mathrm{b}}$ & $20.96^{\mathrm{a}}$ \\
Fósforo (P\%) & $0.27^{\mathrm{a}}$ & $0.21^{\mathrm{a}}$ & $0.22^{\mathrm{a}}$ & $0.39^{\mathrm{a}}$ \\
\hline
\end{tabular}

$a, b$ Letras diferentes en cada fila indican diferencias significativas entre tratamientos $(p<0.05)$

al. (2000) y Fernández et al. (2008) quienes reportaron valores de $43.3,34.2$ y $28.03 \%$, respectivamente para FDA. La NRC (2001), menciona, de otra parte, que los contenidos adecuados para rumiantes de FDA son de $21-27 \%$.

Los contenidos de Ca fueron similares entre tratamientos (Cuadro 4). El resultado obtenido es similar a lo obtenido por Arias
(2015) con $0.21 \%$ para este mineral y al de Salcedo (1998) con $0.39 \%$. Los contenidos de calcio pueden variar dependiendo de la presencia de este mineral en los suelos (Monge et al., 1994). Los valores encontrados en el presente estudio son inadecuados para el mantenimiento de rumiantes de $40 \mathrm{~kg} / \mathrm{p} . \mathrm{v}$, pues la NRC (2001) menciona que los contenidos deben ser de $2.9 \mathrm{~g}$. 
El contenido de fósforo fue mayor en T-4 ( $<<0.05)$ (Cuadro 4). En general, los valores del presente estudio fueron mayores a los hallados por Arias (2015), que encontró promedios de 0.24 y $0.21 \%$ para las avenas Criolla y Mantaro 15, mientras que fueron similares al valor de $0.35 \%$ en el trabajo de Salcedo (1998). Al igual que en el caso del calcio, los niveles de fósforo en la planta pueden variar de acuerdo con los niveles presentes en el suelo, así como por la época de corte y condición ambiental.

\section{Conclusiones}

- La avena Mantaro 15 asociada con vicia obtuvo una mayor producción de materia verde $(>20 \mathrm{t} / \mathrm{ha})$, mientras que los contenidos de materia seca y porcentaje de materia seca fueron similares en los tratamientos.

- En el contenido nutricional, el tratamiento con avena Mantaro 15 asociada con vicia obtuvo mejores resultados de proteína cruda (\%PC); sin embargo, fue similar al tratamiento Mantaro 15 para fibra detergente neutra $(\% \mathrm{FDN})$ y fibra detergente ácida (\%FDA)

- El contenido de fósforo fue mayor en avena Mantaro 15 asociada con vicia que únicamente Mantaro 15.

- Los contenidos de calcio $(\mathrm{Ca} \%)$. Fueron similares entre tratamientos.

\section{Agradecimientos}

A la Universidad Nacional Daniel Alcides Carrión, a través del Vicerrectorado de Investigación, al Instituto Central de Investigación y a los Fondos del Canon y regalías mineras por el financiamiento del presente estudio.

\section{Literatura Cittada}

1. AOAC. 2005. Official methods of analysis of AOAC International, $18^{\text {th }}$ ed. AOAC International. Maryland. USA 80:908-912.
2. Arias AAG. 2015. Estudio de la fenología, rendimiento forrajero, y valor nutritivo de dos variedades de avena (Mantaro 15 y Criolla) en los C.E. Casaracra y Alpaicayan - UNDAC, Papana y Huayllay. Tesis de ingeniero Zootecnista. Pasco, Perú: Univ, Nacional. Daniel Alcides Carrión. 113 p.

3. Ansar M, Ahmed ZI, Malik MA, Nadeem M, Majeed A, Rischkowsky $B A .2010$. Forage yield and quality potential of winter cereal-vetch mixtures under rainfed conditions. Emir J Food Agr 22: 25-36. doi: 10.9755/ejfa.v22i1.4904

4. Britto B. 2017. Actualización de las ecorregiones terrestres de Perú propuestas en el Libro Rojo de Plantas Endémicas del Perú. Gayana Bot 74: 15-29. doi: .10.4067/S0717-66432017005000318

5. Caballero R, Goicoechea EL, Hernaiz PJ. 1995. Forage yields and quality of common vetch and oat sown at varying seeding ratios and seeding rates of vetch. Field Crop Res 41: 135-140. doi: 10.1016/ 0378-4290(94)00114-R

6. Cazares PMJ. 1999. El cultivo de la avena (Avena sativa L). Tesis de Ingeniero Agrónomo Fitotecnista. Saltillo, Coah. Mexico: Univ. Autonoma Agraria Antonio Narro. 67 p.

7. Chávez J, Gómez S. 1999. Guía para producir forraje de avena y cebada bajo temporal en la costa de Ensenada. Instituto Nacional de Investigaciones Forestales, Agrícolas y Pecuarias. Ensenada BC, México.

8. Coblentz WK, Coffey KP, Turner JE, Scarbrough DA, Weyers JS, Harrison $K F$, Johnson ZB, et al. 2000. Effect of maturity on degradation kinetics of sodseeded cereal grain forage grown in northern Arkansas. J Dairy Sci 83: 24992511. doi: 10.3168/jds.S0022-0302(00)75142-3

9. Cocks PS. 1995. Use of pasture and forage legumes in th Mediterranean can increase livestock production and reserve degradation. Diversity 11: $114-155$. 
10. Contreras PJ, De Los Rios B, Montes M, Ramos EY. 2013. Consumo y valor nutricional del ensilado de Calamagrostis antoniana y Avena sativa asociada en diferentes proporciones en alpacas (Vicugna pacos). Rev Complutense Cienc Vet 7: 50-58.

11. Cortes C, Silva M. 1995. Evaluación de híbridos de maíz para ensilajes en la $\mathrm{X}$ región. Resultados de dos temporadas. Avances Prod Anim 20: 229-238

12. Contreras PJ, Ramírez RH, Tunque QM, Aroni QY, Curasma CJ. 2020. Productive and nutritional aspects of forages oats and barley alone and consociated to vetch in high Andean conditions. MOJ Food Process Technol 8: 59-65. doi: 10.15406/mojfpt.2020.08.00243

13. Dumont LJ, Anrique RG, Alomar CD. 2005. Efecto de dos sistemas de determinación de materia seca en la composición química y calidad del ensilaje directo de avena en diferentes estados fenológicos. Agr Tec 65: 388-396. doi: 10.4067/S0365-2807200500040000

14. Espitia RE, Villaseñor HR, Tovar RG, de la Olán M, Limón OA. 2012. Momento óptimo de corte para rendimiento y calidad de variedades de avena forrajera. Rev Mex De Cienc Agric 3: 771- 783.

15. Espinoza MF, Nuñez RW, Ortiz GI, Choque QD. 2018. Producción de forraje y competencia interespecífica del cultivo asociado de avena (Avena sativa) con vicia (Vicia sativa) en condiciones de secano y gran altitud. Rev Inv Vet Perú 29: 1237-1248. doi: 10.15381/rivep.v29i4.15202

16. Fernández A, Lagranje S, Bolleta A, Tulesi M, Larrea D. 2008. Evaluación de calidad nutricional de diferentes estados de madurez del cultivo de avena para la obtención de henos o silaje de planta entera. INTA Bordenave, Argentina. $12 \mathrm{p}$.

17. Flores NM, Sánchez GR, Echavarría CF, Gutiérrez, LR, Rosales NC, Salinas GH. 2016. Producción y calidad de forraje en mezclas de veza común con cebada, avena y triticale en cuatro etapas fenológicas. Rev Mex Cienc Pecu 7:275-291. doi: 10.22319/rmcp.v7i3.4210

18. Florián R. 2006. Evaluación del rendimiento y composición química de la asociación avena-vicia forrajeros en Cajamarca. En: XXIX Reunión de la Asociación Peruana de Producción Animal. Huancayo, Perú.

19. Garduño-Castro Y, Espinoza-Ortega $A$, González-Esquivel CE, MateoSalazar B, Arriaga-Jordán CM. 2009. Intercropped oats (Avena sativa) common vetch (Vicia sativa) silage in the dry season for small-scale dairy systems in the highlands of central Mexico. Trop Anim Health Pro 41: 827834. doi: $10.1007 / \mathrm{s} 11250-008-9258-7$

20. Giller KE. 2001. Nitrogen fixation in tropical cropping systems. Wallingford, UK: CABI Publishing. $448 \mathrm{p}$.

21. Graham PH, Vance CP. 2000. Nitrogen fixation in perspective: an overview of research and extension needs. Field Crops Res 65: 93-106. doi: 0.1104/ pp.017004

22. Johnston J, Wheeler B, Mckinlay J. 1999. Forage production from spring cereals and cereal-pea mixtures. Ministry of Agriculture, Food and Rural Affairs. No. 120. Ontario, Canada. 229 p.

23. Kim TS, Raveendar S, Suresh S, Lee GA, Lee JR, Cho JH, Lee SY, et al. 2015. Transcriptome analysis of two $\mathrm{Vi}$ cia sativa subspecies: mining molecular markers to enhance genomic resources for vetch improvement. Genes 6: 11641182. doi: $10.3390 /$ genes 6041164

24. Lithourgidis AS, Vasilakoglou IB, Dhima KV, Dordas CA, Yiakoulaki MD. 2006. Forage yield and quality of common vetch mixtures with oat and triticale in two seeding ratios. Field Crop Res 99: 106-113. doi: 10.1016/ j.fcr.2006.03.008

25. Monge E, Val J, Sanz M, Blanco A, Montañez L. 1994. Calcium as a nutrient for plants. The bitter pit in apple. An Estac Exp Aula Dei 21: 189-201. 
26. Noli E, Canto A, Segura J. 2006. La avena forrajera INIA Mantaro 15 mejorado una alternativa de forraje para la zona altoandina. En: Reunión de la Asociación Peruana de Producción Animal. Huancayo, Perú.

27. Noli HEC, Peralta TE, Ordoñez FH, Nestares PA. 2010. Caracterización agronómica en avena forrajera para producción semilla en la sierra central del Perú. En: XXXII Reunión Anual de Asociación Peruana de Producción Animal. Lima, Perú.

28. Noli C, Asto R, Canto A. 2004a. Evaluación de variedades de avena forrajera tolerantes a sequias y heladas para la producción de forraje verde. Instituto Nacional de Investigación y Extensión Agraria - INIEA. [Internet]. Disponible en: http://repositorio.inia.gob.pe/handle/ 20.500.12955/594

29. Noli C, Segura J, Canto A. 2004b. La avena forrajera INIA mantaro 15 mejorada una alternativa de forraje para la zona altoandina. Instituto Nacional de Investigación y Extensión Agraria INIEA. [Internet]. Disponible en: http:// repositorio.inia.gob.pe/handle/ 20.500.12955/590

30. NRC. 2001. Nutrient requirements of dairy Cattle. $7^{\text {th }}$ ed. National Research Council. National Academy of Sciencie. Washington DC, USA. 381 p.

31. Oliver AL, Grant RJ, Pedersen JF, O'Rear J. 2004. Comparison of brown midrib-6 and-18 forage sorghum with conventional sorghum and corn silage in diets of lactating dairy cows. J Dairy Sci
87: 637-644. doi: 10.3168/jds.S00220302(04)73206-3

32. Ramírez OS, Domínguez DD, Salmerón ZJJ, Villalobos VG, Ortega GJA. 2013. Producción y calidad del forraje de variedades de avena en función del sistema de siembra y de la etapa de madurez al corte. Rev Fitotec Mex 36: 395-403.

33. Salcedo G. 1998. Valor nutritivo y degradabilidad ruminal de la Avena sativa y Vicia sativa. Pastos 28: 71-85.

34. Shin DE, Kim DA, Choi KJ, Kim WH. 1999. Forage yield and quality of oats as affected by different types and $\mathrm{N}$ rates of liquid Manure. J Korean Grassl Sci 19: 121-126. doi: 10.4236/ ajps.2015.619316

35. Salmerón ZJJ, Meda FJ, Barcena JR. 2003. Variedades de avena y calidad nutricional del forraje. Folleto Técnico $\mathrm{N}^{\circ}$ 17. CESICH-CIRNOC-INIFAP-SAGARPA. Chihuahua, México. $43 \mathrm{p}$.

36. Villareal N. 2013. Evaluación de tres dietas alimenticias a base de llantén forrajero (Plantago lanceolata), maíz (Zea mays) y avena forrajera (Avena sativa), para la ganancia de peso en cuyes en etapa de crecimiento. Tesis de Ingeniero en Desarrollo Integral Agropecuario. Tulcán, Ecuador: Univ. Politécnica Estatal del Carchi. 71 p.

37. Zapana PJG, Miranda CF, Villalta RP. 2014. Producción de semilla de avena forrajera (Avena sativa L.) con incorporación de humus de lombriz en el centro de investigación y producción Camacani. Puno. Perú. Rev Investig Altoandin 16: 39-42. 